\title{
Temperature Dependence of Whispering Gallery Modes of Quantum Dot-Doped Microbottle Resonators
}

\author{
Shubhayan Bhattacharya ${ }^{1)}$, Aneesh V. Veluthandath ${ }^{1)}$, Ganapathy Senthil Murugan ${ }^{2)}$ and Prem B. Bisht ${ }^{\left.1^{*}\right)}$ \\ 1) Department of Physics, Indian Institute of Technology, Madras, Chennai India - 600036 \\ 2) Optoelectronics Research Centre, University of Southampton, Southampton, UK
}

E-mail: bisht@iitm.ac.in

Received xxxxxx

Accepted for publication $\mathrm{xxxxxx}$

Published xxxxxx

\begin{abstract}
The temperature dependence of the whispering gallery modes (WGMs) of self-assembled microbottle resonators (MBRs) made of poly (methyl methacrylate) (PMMA) have been studied. The MBRs are doped with fluorescent cadmium selenide quantum dots (QDs). WGMs of MBRs are found to exhibit a blue shift on increasing the temperature over a wide temperature range. It has been observed that the shifts are linear with temperature over $\mathrm{a} \sim 10 \mathrm{~nm}$ wavelength range studied here. The observed shifts are stable over several cycles and longer exposure times indicating high photo-stability.
\end{abstract}

Keywords: Microbottle resonator; whispering gallery modes; fluorescence based sensor; polymer; quantum dot

\section{Introduction}

Dielectric microresonators have applications as microlasers [1-3], micro-sensors [4,5] and in frequency stabilization [6]. The symmetric, dielectric resonators give rise to whispering gallery modes (WGMs) in the electromagnetic spectrum. Geometries such as circular [7], rectangular [8], spherical [9] and microtoroids [10] have been extensively studied. However, microbottle resonators (MBRs) are relatively new in literature [11]. In contrast to the spheres or toroids, the modes in MBRs are extended along the axis of the resonator. The free spectral range (FSR) of MBRs is an order of magnitude smaller than that of the microspheres of same dimension [12]. This is due to the fact that the light ray in MBRs traverses a longer path - an added advantage for improved light-interrogation with measurands for sensing applications. Among a few methods of preparing MBRs $[11,13]$, the self-assembly process is simpler and easier to fabricate quickly in large numbers. Recently, the selfassembled MBRs supporting Q values of the order $10^{6}$ have been reported [14].
Quantum dots (QDs) are semiconductor particles with sizes less than the exciton Bohr radius. Their optical properties lie in between that of the bulk and a single molecule [15]. The optical, photoluminescence (PL) and electronic properties of QDs can be tuned by varying their sizes. One of the popularly studied semiconductor QDs is cadmium selenide (CdSe). The CdSe QDs have band gap in the range of 1.7-2.1 eV. These have been used in optoelectronic devices, solar cells [16] and light emitting diodes applications [17].

Since WGMs are highly sensitive to the size and refractive index of the resonator, any change in these parameters causes a shift in the position of a mode. Temperature dependent studies of bare microresonators and doped with dyes have been reported in literature [18-21]. The photobleaching of dyes is known to induce errors in measurement [21]. To avoid photobleaching, inorganic fluorophores such as QDs of CdSe with high photobleaching threshold can be used to prepare self-assembled MBRs (sMBRs). Polymethyl methacrylate (PMMA) can be used due to its good optical transparency in the visible and near-infrared wavelengths [22]. In this paper, finite element modelling has been used to mimic the 
experimental system to understand the amount of shift in WGMs for the temperature range of $-50{ }^{\circ} \mathrm{C}$ to $75{ }^{\circ} \mathrm{C}$. The experiments are performed on the temperature dependence of CdSe QD doped PMMA sMBRs with free-space pumping. It is observed that the simulated and experimental data are in good agreement and the system has high photo- and temporal stability for probable applications in sensing.

\section{Experimental Details}

$\mathrm{CdSe} / \mathrm{CdS}$ core shell QDs dispersed in hexane were obtained from CANdots. PMMA (average molecular wt. 120,000) and toluene $(99.8 \%)$ were obtained from Sigma-Aldrich. The tapered fibers were made by heat and pull method using a standard single mode optical fiber [11]. The sMBRs were prepared by a method described elsewhere [14]. Briefly, a tapered fiber was dipped in the PMMA solution of toluene and withdrawn. During this process, a thin layer of PMMA gets coated on the surface of the cylindrical tapered fiber. The coated cylindrical fiber was kept in vertical position such that the excess of PMMA moves down the fiber while the toluene evaporates. Subsequently the coated PMMA solution breaks into several small segments and forms bottle-like shapes due to surface tension [14]. It was observed that the shape of the microbottles remained unaltered during the evaporation of the solvent. The sample was further baked at $110{ }^{\circ} \mathrm{C}$ in the oven to dry the PMMA completely. CdSe QD doped sMBRs could be prepared by just dissolving QDs in the PMMA solution and following the above mentioned procedure.

The PL measurements were done using a high resolution $(0.02 \mathrm{~nm})$ micro Raman spectrometer (Horiba Jobin Yvon, HR800UV) on excitation at $488 \mathrm{~nm}$ (Fig. 1). The laser power was kept at $0.1 \mathrm{~mW}$ to avoid laser heating and the time of exposure was kept at 30s. The sMBR was placed inside a temperature controlled $\left( \pm 0.01{ }^{\circ} \mathrm{C}\right)$ sample chamber (LINKAM, THMS 600) with only its stems supported by silicon substrates such that the bottle region is sitting freely as shown in Panel B of Fig. 1. To achieve lower temperatures, liquid nitrogen was constantly pumped in to the chamber. The temperature was varied at a rate of $5{ }^{\circ} \mathrm{C} / \mathrm{min}$ and the sample was allowed to thermalise for 10 minutes before taking a PL measurement.

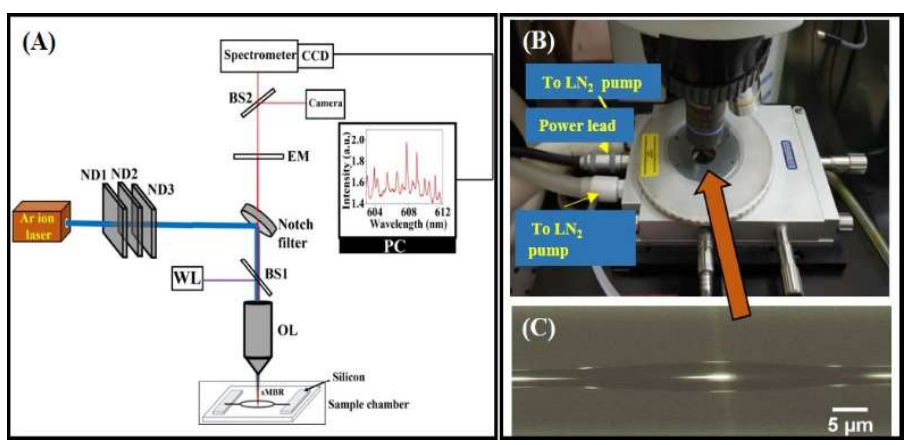

Fig. 1. Schematic of the measurement setup (panel A). ND- neutral density filters, BS- beam splitter, OL-objective lens, EM- emission filter and PC- personal computer. The fiber stems of the sMBR were placed on silicon substrates in the temperature-controlled sample chamber (B) mounted on the microscope stage. The thick arrow indicates the place inside the sample chamber where the sMBR (C) is mounted.

\section{Modelling of the PMMA bottle resonator}

The MBR has been modelled to look into the behavior of the resonance frequency of a particular bottle mode as a function of the temperature. Fig. $2 \mathrm{~A}$ shows the schematic of an sMBR. The blue lines indicate the trajectory of the bottle modes. The resonant modes are identified by three quantum numbers $(m$, $p, q)$ where $m$ is the azimuthal quantum number or mode number, $p$ is the radial quantum number and $q$ is the axial quantum number.

The finite element modelling (Comsol Multiphysics 5.2) was used to simulate the WGMs of an MBR with dimensions $\mathrm{D}_{\mathrm{b}}=5 \mu \mathrm{m}, \mathrm{D}_{\mathrm{s}}=2 \mu \mathrm{m}$ and $\mathrm{L}_{\mathrm{b}}=30 \mu \mathrm{m}$. The electric field distribution of a typical mode $\mathrm{TE}_{70,1,1}$ is shown in Fig. 2(B). The shifts due to thermal effects on the WGMs of a microcavity appear due to two factors, (i) linear expansion coefficient of the material $(\delta)$, and (ii) its thermo-optic coefficient $(\beta)$. The net shift of a WGM is related to the shifts due to $\delta$ and $\beta$ by the following relation [23]

$$
\Delta \lambda=\lambda(\delta+\beta) \Delta T
$$

where $\delta=\frac{1 d D_{b}}{D_{b} d T}, \beta=\frac{1 d n}{n d T}$ and $\Delta \mathrm{T}$ is the change of temperature of the microcavity and $\mathrm{n}$ is the refractive index of the material (PMMA). 


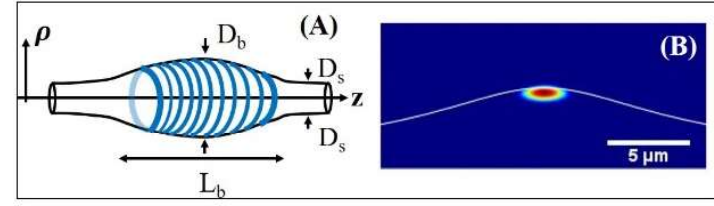

Refractive index

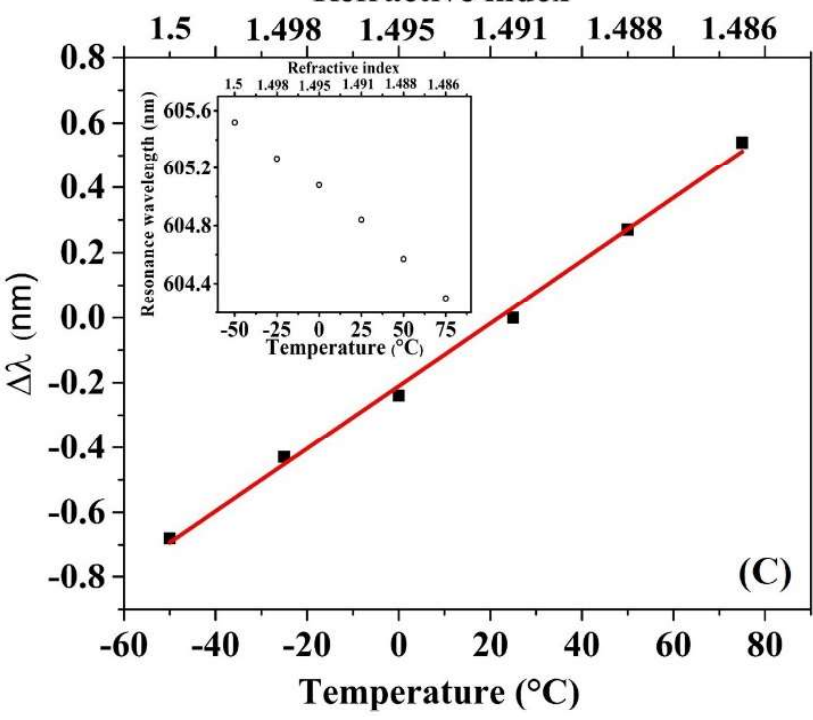

Fig. 2. (A) Schematic of the sMBR. $D_{b}$ - bottle diameter, $D_{s^{-}}$stem diameter and $\mathrm{L}_{\mathrm{b}}$ - length of the bottle resonator. (B) shows the calculated fundamental bottle mode $\left(\mathrm{TE}_{70,1,1}\right)$ and $(\mathrm{C})$ shows $\Delta \lambda$ as a function of temperature. Inset shows the behavior of the position of the mode as a function of temperature.

The effect $\delta$ and $\beta$ on the MBR was studied as a function of temperature. The reported value of $\delta$ obtained by thermal interferometry [24] have been used for PMMA. The WGM shift due to $\beta$ were calculated from $n=1.5$ (at $-50{ }^{\circ} \mathrm{C}$ ) to $\mathrm{n}=1.486$ (at $75{ }^{\circ} \mathrm{C}$ ) [25]. It is observed that the peak wavelength of the mode decreases with increase in temperature indicating a blue shift (see inset, Fig. 2C). The net shift has been calculated to be $1.22 \mathrm{~nm}$ for the temperature range of $125^{\circ} \mathrm{C}$.

\section{Results and Discussion}

\subsection{Steady state absorption and PL spectra}

The absorption spectrum of the colloidal solution of CdSe QD in hexane shows two humps (at $472 \mathrm{~nm}$ and $600 \mathrm{~nm}$ ) corresponding to its first excitonic peaks (Fig. 3A). The PL spectrum shows a maximum at $610 \mathrm{~nm}$. These values are in agreement to those reported in literature. A Tauc plot [26] has been used for estimating the energy band gap of $\mathrm{CdSe} / \mathrm{CdS}$ core shell QDs suspended in hexane as follows. For a direct band gap semiconductor $(\alpha h v)^{2}$ is plotted as a function of energy ( $h v)$ where $\alpha$ is the absorption coefficient that can be estimated from the optical density (OD) using the relation $\alpha=2.303 \times O D / d$ where $d$ is the thickness of the sample. A straight line is fitted on the plotted curve. The $\mathrm{x}$-intercept of the fitted straight line gives the band gap of $1.97 \mathrm{eV}$ (Fig. 3B).
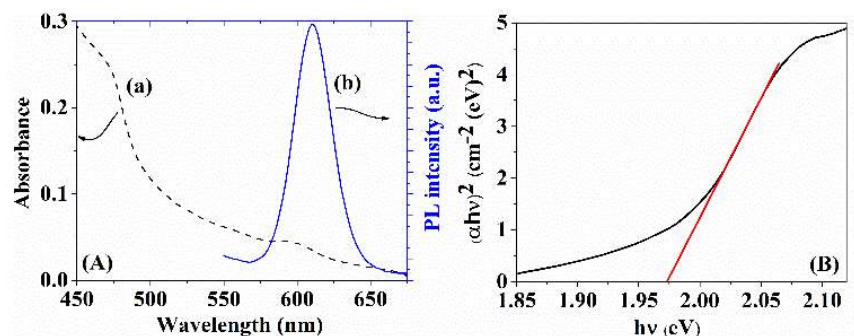

Fig. 3. Panel A. Absorption (a) and PL (b) spectrum $\left(\lambda_{\mathrm{exc}}=488 \mathrm{~nm}\right)$ of CdSe QDs in hexane. Panel B. Tauc plot of the CdSe QD in hexane. Here $\alpha, v$ and $h$ denotes the absorption coefficient, frequency and Planck's constant respectively.

Fig. 4 shows the room temperature PL spectra of two selfassembled CdSe QD doped PMMA microbottle resonators of $\mathrm{D}_{\mathrm{b}}-5 \mu \mathrm{m}$ (sMBR1) and $6 \mu \mathrm{m}$ (sMBR2), respectively with a same $D_{s}$ of $2 \mu \mathrm{m}$. The spectra exhibit series of repetitive peaks built upon the broad PL background of CdSe QD. Such peaks are assigned to the WGMs of the microbottle resonator [27]. The number of peaks in the spectra increases with increase in size of the microbottle. This is due to the fact that the value of FSR increases on decreasing the diameter of the SMBR [12]. The FSR for sMBR1 and sMBR2 are $1.13 \mathrm{~nm}$ and $0.83 \mathrm{~nm}$, respectively.

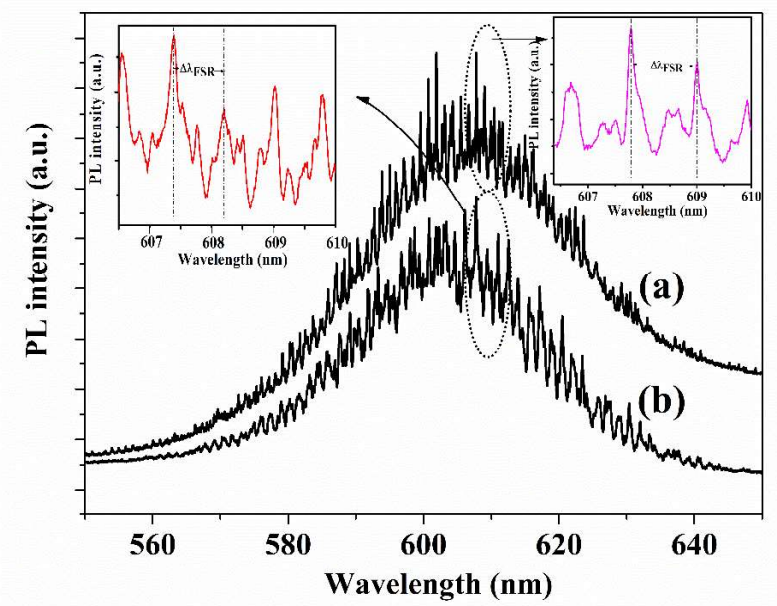

Fig. 4. Experimentally observed WGMs in PL of CdSe doped (a) sMBR1 $\left(D_{b}=5 \mu \mathrm{m}\right)$ and (b) sMBR2 $\left(D_{b}=6 \mu \mathrm{m}\right)$. The values of $D_{s}$ $\sim 2 \mu \mathrm{m}$ and $\mathrm{L}_{\mathrm{b}}(30 \mu \mathrm{m})$ are about same for both the sMBRs. $\Delta \lambda_{F S R}$ is indicated in the expanded portions of the spectra (insets).

\subsection{Experimentally observed WGM shift with temperature}

Fig. 5 shows the typical temperature dependent PL spectra of sMBR 1 and sMBR2 for a temperature range of $125^{\circ} \mathrm{C}$. As can be seen the WGM peaks consistently shift towards the shorter wavelength on increasing the temperature. In a perfect 
microsphere, the effect of thermal expansion is only due to the equatorial plane with dominant contribution of the fundamental mode. In elongated microresonators such as MBR, changes in both the diameter and length of the MBR may affect the modes. The MBR resonator profile is an approximate parabola given by [11]

$$
D(z) \approx D_{b}\left(1-\frac{1}{2}(\Delta k z)^{2}\right)
$$

where $R_{0}$ is the maximum value of the radius at the center of $\operatorname{MBR}(z=0)$ and $\Delta k$ is the curvature of the bottle profile. The value of $\delta$ for a bottle resonator can be obtained by differentiating eq. 2 with respect to $\mathrm{T}$ as

$$
\delta=\frac{1 d D(z)}{D(z) d T}
$$

The value of $D(z)$ at the measurement position $(\mathrm{z}=0)$ is $\mathrm{D}_{\mathrm{b}}$, eq. 1 follows the trend similar to that for the microspheres. Since $\delta=79.6 \times 10^{-6} /{ }^{\circ} \mathrm{C}$ and $\beta \sim-10^{-4}$ [24], eq. 1 shows that the thermal refraction of PMMA causes a blue-shift of the WGMs while the thermal expansion causes a red-shift for $\Delta T>0$. Since $\beta$ is negative and its absolute value is larger than $\delta$, a net blue-shift is observed with temperature in the present case. This shift in the resonance wavelength of the WGMs observed as a function of temperature can act as the parameter for sensing.

The size parameter $(x=2 \pi r / \lambda)$ is smaller for longer wavelengths, and hence the resonance shifts are non-uniform and the values increase towards the red for smaller MBR. This is due to the fact that various bottle modes have different optical paths resulting in non-uniform resonant shifts. For a temperature range of $125^{\circ} \mathrm{C}$, for example, resonant shifts for resonance peak I, II and III are $1.10,1.12$ and $1.15 \mathrm{~nm}$, respectively for sMBR1 (Fig. 5, Panel A), while the maximum spectral blue-shift for sMBR2 (for resonance peak III) is 0.99 $\mathrm{nm}$. The resonant shifts have been summarized in Table 1. It should be noted that the measured $\Delta \lambda$ follows linear relation with T (see Fig. 6, Panel A).

Table 1 Summary of the maximum shifts of resonance peaks for the $s M B R s$

\begin{tabular}{|l|c|c|c|}
\hline \multirow{2}{*}{ Bottle } & \multicolumn{3}{|c|}{ Shifts (nm) } \\
\cline { 2 - 4 } & Peak I & Peak II & Peak III \\
\hline sMBR1 & 1.10 & 1.12 & $1.15^{*}$ \\
sMBR2 & 0.95 & 0.98 & 0.99 \\
\hline
\end{tabular}

* modelled maximum shift has been found to be $1.22 \mathrm{~nm}$ for sMBRl
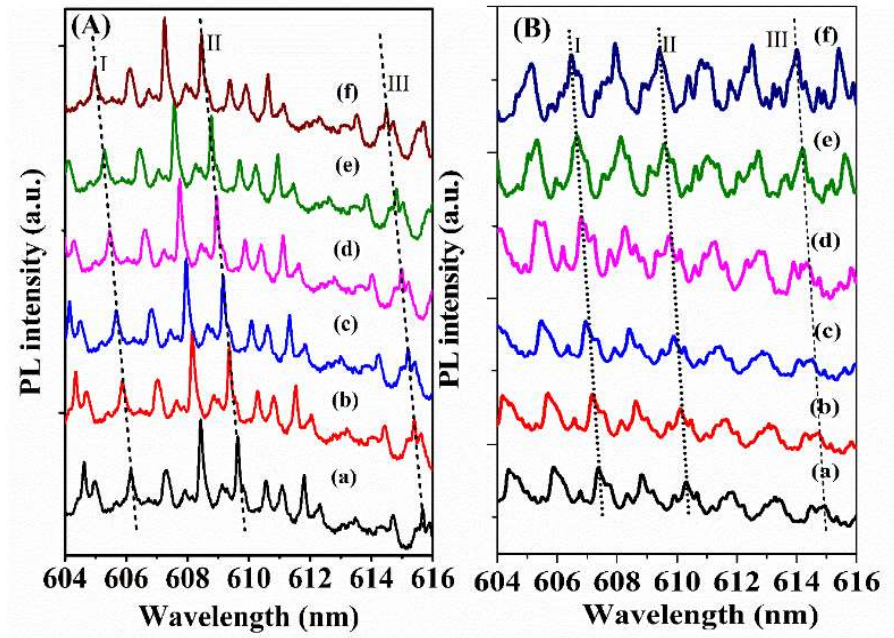

Fig 5. Temperature dependence of the WGMs in sMBR1 (Panel A) and sMBR2 (Panel B) in the $610 \mathrm{~nm}$ band. The temperatures are (a) $-50{ }^{\circ} \mathrm{C}$, (b) $-25^{\circ} \mathrm{C}$, (c) $0{ }^{\circ} \mathrm{C}$, (d) $25^{\circ} \mathrm{C}$, (e) $50{ }^{\circ} \mathrm{C}$, and (f) $75^{\circ} \mathrm{C}$. Slanted dashed lines are guide to the eye.

\subsection{Photo- and temporal stability of CdSe QD doped $S M B R$}

To check the photo-stability and temporal stability of the system at a low power of $0.1 \mathrm{~mW}$, two experiments were performed on sMBR2. The PL measurement was repeated every 10 mins at $25^{\circ} \mathrm{C}$ at the identical experimental conditions while the sample is repeatedly exposed to the excitation beam. Fig. 6, Panel B shows the PL intensity recorded at 0 and 120 mins during the exposure. It can be seen that the PL intensity is unaffected even after 120 mins of exposure with the excitation light. It should also be noted that the mode positions remain unchanged with time. In this sense, the stability of the QD doped MBRs is better than that of dye doped WGM sensors [21].

For checking the temporal stability and repeatability of the sMBR temperature sensor, another experiment was performed by varying the temperature between $0{ }^{\circ} \mathrm{C}$ and $50{ }^{\circ} \mathrm{C}$ and observing the spectral shifts of a particular mode as a function of time as follows. Initially, the temperature is brought down to $0{ }^{\circ} \mathrm{C}$ and four PL spectra were recorded consecutively at a time interval of 10 mins. It was observed that the position and the intensity of the WGMs do not show any appreciable change. Subsequently, the temperature was brought up at $50{ }^{\circ} \mathrm{C}$ and was stabilized for 10 minutes before the measurements. At each temperature again, the PL spectra were recorded at the time interval of 10 mins. The total time of the measurement was 150 mins. The WGM shift was monitored with reference to the resonance wavelength obtained at $25{ }^{\circ} \mathrm{C}$ as shown in the inset of Fig. 6, Panel B. It can be seen that the WGM shift remains constant and 
reproducible at a particular temperature. The effect of nonradiative processes which may cause some degree of heating does not seem to affect the positions of the WGMs.
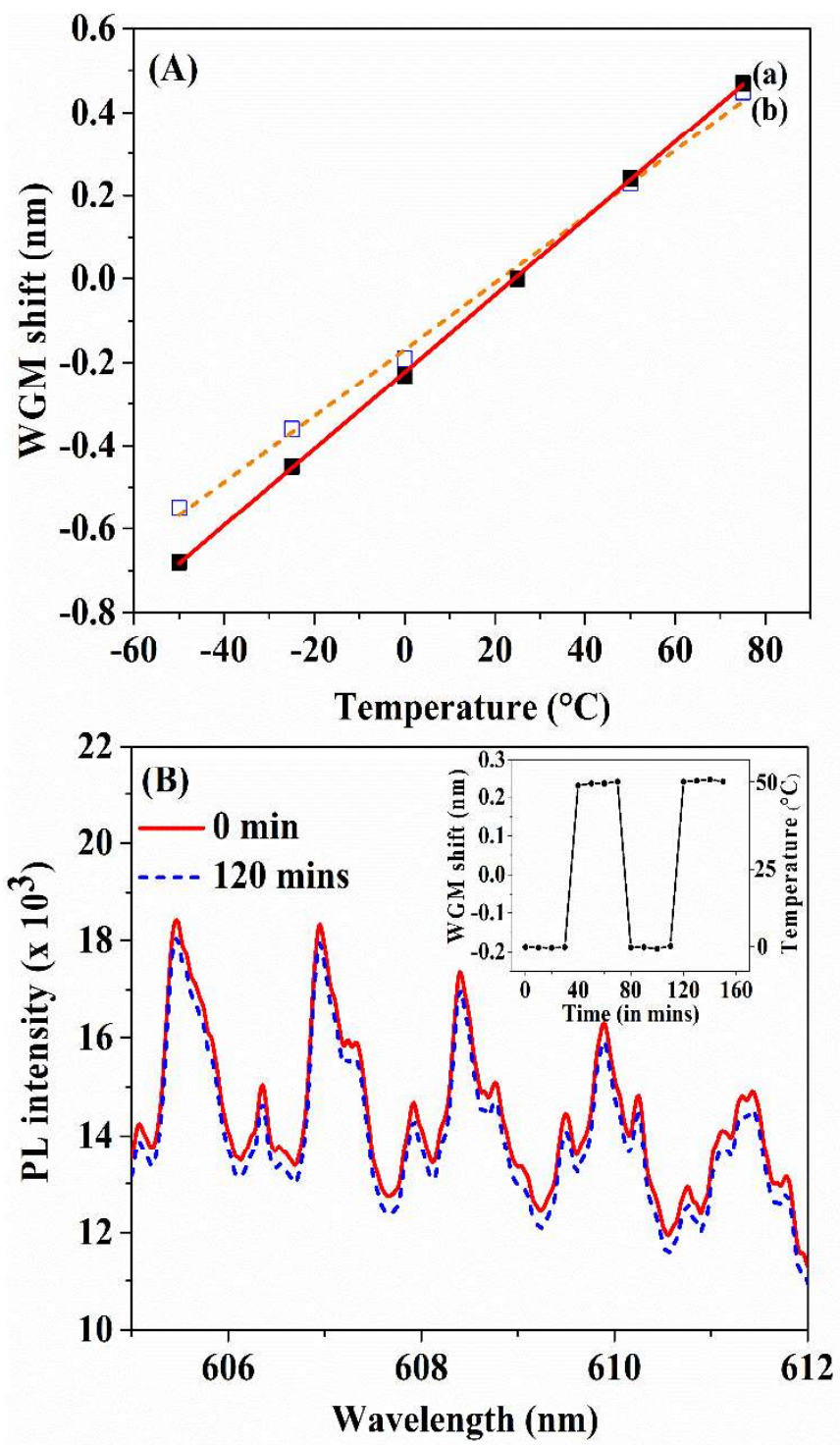

Fig 6. Panel A: Measured WGM shifts for sMBR1 ( $\square$ (a) and SMBR2 (ㅁ) (b) as a function of temperature. The solid and dashed lines indicate the linear fits to the experimental data. Panel B: WGMs in the PL spectra of sMBR2 at different repeated exposure times as indicated in the figure. Inset shows the WGM shifts as a function of time at two different temperatures $0{ }^{\circ} \mathrm{C}$ and $50{ }^{\circ} \mathrm{C}$.

\subsection{Sensitivity of the device}

Sensitivity (S) of a temperature sensor is the ratio of the variation of the wavelength $(\Delta \lambda)$ with the change in temperature $(\Delta T)$. The maximum spectral shift from modelling has been found to be $1.22 \mathrm{~nm}$ and thus the calculated value of $\mathrm{S}$ is found to be higher $\left(9.4 \mathrm{pm} /{ }^{\circ} \mathrm{C}\right)$ than that measured experimentally for $\operatorname{sMBR} 1\left(9.2 \mathrm{pm} /{ }^{\circ} \mathrm{C}\right)$ and
sMBR2 (7.9 pm/ $\left.{ }^{\circ} \mathrm{C}\right)$. The highest sensitivity has been obtained to be $9.2 \mathrm{pm} /{ }^{\circ} \mathrm{C}$, thus estimating the resolution $\left(\Delta \mathrm{T}_{\min }=\left(\Delta \lambda_{\min } /(\mathrm{d} \lambda / \mathrm{dT})\right)[18]\right.$ of the sMBR temperature sensor to be $2{ }^{\circ} \mathrm{C}$. It was observed that the sensitivity was higher for the smaller sMBR (sMBR1) similar to that obtained for air pressure sensing with silica microspheres [28]. This is due to the fact that the size parameter and hence $\Delta \mathrm{x}$ is smaller for resonators with smaller sizes resulting in larger $\Delta \lambda$. This is supported by simulations for a PMMA cylindrical microresonator, where it is observed that the $\Delta \lambda$ is larger for a smaller cylindrical resonator (see supplementary information).

\subsection{Deviation parameter}

To account for the variation of the experimental and calculated $\mathrm{S}$, we include a deviation parameter $\gamma$ in eq. 1 such that

$$
\Delta \lambda^{\prime}=\lambda(\delta+\beta+\gamma) \Delta T
$$

The average value of $\gamma$ has been found to be $0.6 \pm 0.4 \times$ $10^{-6} /{ }^{\circ} \mathrm{C}$. The appearance of $\gamma$ can be ascribed to the following two factors. (i) as the MBR is prepared on the tapered fiber made of silica, the positive value of $\gamma$ is assigned to the red-shift due to silica on the WGM resulting in a net blue shift and (ii) the value of $\delta$ and $n$ used [24,25] for calculating the wavelength shift may be within the accuracy of value of $\gamma$ in the present case.

Even though the sensitivity is low in this case compared to liquid crystal (LC) microdroplets based temperature sensor [21], the present system is highly photostable. In case of LC microdroplets, the thermal expansion coefficient is greater than $10^{-3} /{ }^{\circ} \mathrm{C}[21]$, more than a magnitude higher than that of PMMA, thus resulting in higher sensitivity. Therefore, CdSe QD incorporated in LC based microresonators will result in highly sensitive and photostable temperature sensor.

\section{Conclusions}

QD doped self-assembled microbottle resonators exhibit WGMs in the luminescence spectra. The effect of temperature has been studied from $-50{ }^{\circ} \mathrm{C}$ to $75{ }^{\circ} \mathrm{C}$. The WGMs show a linear blue-shift with increase in the temperature. It has been observed that the smaller sMBR shows higher sensitivity with temperature compared to the larger one. The shift in the positions of the WGMs in sMBRs with change in temperature along with the high photo-stability of the system can be used in fluorescence based temperature sensor applications. 


\section{Supplementary files}

The higher sensitivity of smaller resonator is given as supplementary information.

\section{Acknowledgements}

The authors thank Department of Science and Technology (DST), New Delhi, for financial assistance. PBB thanks financial support by IITM grant no.

TTR1819015IITMPREM.

\section{Disclosures}

The authors declare no conflict of interest.

A. V. Veluthandath, S. Bhattacharya, G.S. Murugan, P.B. Bisht, Fano resonances and photoluminescence in selfassembled high-quality-factor microbottle resonators, IEEE Photonics Technol. Lett. 31 (2019) 226-229. doi:10.1109/LPT.2018.2889433.

11925. doi:10.1364/OE.17.011916.

Y. Louyer, D. Meschede, A. Rauschenbeutel, Tunable whispering-gallery-mode resonators for cavity quantum electrodynamics, Phys. Rev. A. 72 (2005) 031801 1-4. doi:10.1103/PhysRevA.72.031801.

M. Pöllinger, D. O'Shea, F. Warken, A. Rauschenbeutel, Ultrahigh-Q Tunable Whispering-Gallery-Mode Microresonator, Phys. Rev. Lett. 103 (2009) 053901 1-4. doi:10.1103/PhysRevLett.103.053901.

E. Purcell, H. Torrey, R. Pound, Resonance Absorption by Nuclear Magnetic Moments in a Solid, Phys. Rev. 69 (1946) 37-38. doi:10.1103/PhysRev.69.37.

[16] I. Robel, V. Subramanian, M. Kuno, P. V. Kamat, Quantum dot solar cells. Harvesting light energy with CdSe nanocrystals molecularly linked to mesoscopic TiO2films, J. Am. Chem. Soc. 128 (2006) 2385-2393. doi:10.1021/ja056494n.

[1] M. Cai, O. Painter, K.J. Vahala, P.C. Sercel, Fiber-coupled microsphere laser., Opt. Lett. 25 (2000) 1430-1432. doi:10.1364/OL.25.001430.

[2] G.S. Murugan, M.N. Zervas, Y. Panitchob, J.S. Wilkinson, Integrated Nd-doped borosilicate glass microsphere laser., Opt. Lett. 36 (2011) 73-5. doi:10.1364/OL.36.000073.

[3] T. Reynolds, N. Riesen, A. Meldrum, X. Fan, J.M.M. Hall, T.M. Monro, A. François, Fluorescent and lasing whispering gallery mode microresonators for sensing applications, Laser Photonics Rev. 11 (2017) 1-20. doi:10.1002/lpor.201600265.

[4] V.R. Dantham, S. Holler, C. Barbre, D. Keng, V. Kolchenko, S. Arnold, Label-Free Detection of Single Protein Using a Nanoplasmonic-Photonic Hybrid Microcavity, Nano Lett. 13 (2013) 3347-3351. doi:10.1021/nl401633y.

[5] M. Himmelhaus, A. Francois, In-vitro sensing of biomechanical forces in live cells by a whispering gallery mode biosensor, Biosens. Bioelectron. 25 (2009) 418-427. doi:10.1016/j.bios.2009.07.021.

[6] S. Schiller, R.L. Byer, High-resolution spectroscopy of whispering gallery modes in large dielectric spheres., Opt. Lett. 16 (1991) 1138-1140. doi:10.1364/OL.16.001138.

[7] C.P. Dettmann, G. V. Morozov, M. Sieber, H. Waalkens, Unidirectional emission from circular dielectric microresonators with a point scatterer, Phys. Rev. A. 80 (2009) 063813 1-11. doi:10.1103/PhysRevA.80.063813.

[8] M. Mendez-Astudillo, H. Okayama, H. Nakajima, Evanescently Coupled Rectangular Microresonators in Silicon-on-Insulator with High Q-Values: Experimental Characterization, Photonics. 4 (2017) 1-12. doi:10.3390/photonics4020034.

[9] A. Chiasera, Y. Dumeige, P. Féron, M. Ferrari, Y. Jestin, G.N. Conti, S. Pelli, S. Soria, G.C. Righini, Spherical whispering-gallery-mode microresonators, Laser Photonics Rev. 4 (2010) 457-482. doi:10.1002/lpor.200910016.

[10] S.M. Spillane, T.J. Kippenberg, K.J. Vahala, K.W. Goh, E. Wilcut, H.J. Kimble, Ultrahigh-Q toroidal microresonators for cavity quantum electrodynamics, Phys. Rev. A. 71 (2005) 013817 1-10. doi:10.1103/PhysRevA.71.013817.

[11] G. Senthil Murugan, J.S. Wilkinson, M.N. Zervas, Selective excitation of whispering gallery modes in a novel
[17] V.L. Colvin, M.C. Schlamp, A.P. Alivisatos, Lightemitting diodes made from cadmium selenide nanocrystals and a semiconducting polymer, Nature. 370 (1994) 354357. doi:10.1038/370354a0. silica micro-resonators, J. Phys. D. Appl. Phys. 41 (2008) 245111. doi:10.1088/0022-3727/41/24/245111.

[19] L. Wan, H. Chandrahalim, C. Chen, Q. Chen, T. Mei, Y. Oki, N. Nishimura, L.J. Guo, X. Fan, On-chip, highsensitivity temperature sensors based on dye-doped solidstate polymer microring lasers, Appl. Phys. Lett. 111 (2017) 061109 1-5. doi:10.1063/1.4986825.

[20] Y.-Z. Yan, C.-L. Zou, S.-B. Yan, F.-W. Sun, Z. Ji, J. Liu, Y.-G. Zhang, L. Wang, C.-Y. Xue, W.-D. Zhang, Z.-F. Han, J.-J. Xiong, Packaged silica microsphere-taper coupling system for robust thermal sensing application, Opt. Express. 19 (2011) 5753-5759. doi:10.1364/OE.19.005753.

[21] Z. Liu, L. Liu, Z. Zhu, Y. Zhang, Y. Wei, X. Zhang, E. Zhao, Y. Zhang, J. Yang, L. Yuan, Whispering gallery mode temperature sensor of liquid microresonastor, Opt. Lett. 41 (2016) 4649-4652. doi:10.1364/OL.41.004649.

[22] M.A. Khashan, A.Y. Nassif, Dispersion of the optical a wide spectral range: $0.2-3 \mu \mathrm{m}$, Opt. Commun. 188 (2001) 129-139. doi:10.1016/S0030-4018(00)01152-4.

[23] C.H. Dong, L. He, Y.F. Xiao, V.R. Gaddam, S.K. Ozdemir, Z.F. Han, G.C. Guo, L. Yang, Fabrication of high- Q polydimethylsiloxane optical microspheres for thermal sensing, Appl. Phys. Lett. 94 (2009) 1-4. doi:10.1063/1.3152791.

[24] J.A. Corsetti, W.E. Green, J.D. Ellis, G.R. Schmidt, D.T. Moore, Measurement of linear coefficient of thermal expansion and temperature-dependent refractive index using interferometric system, in: Opt. Des. Fabr. 2017 (Freeform, IODC, OFT), OSA, Washington, D.C., 2017: p. OW4B.3. doi:10.1364/OFT.2017.OW4B.3.

[25] J.M. Cariou, J. Dugas, L. Martin, P. Michel, Refractiveindex variations with temperature of PMMA and polycarbonate., Appl. Opt. 25 (1986) 334-336. doi:10.1364/AO.25.000334.
[18] Q. Ma, T. Rossmann, Z. Guo, Temperature sensitivity of constants of quartz and polymethyl methacrylate glasses in 
[26] J. Tauc, R. Grigorovici, A. Vanou, Optical Properties and Electronic Structure of Amorphous Germanium, Phys. Status Solidi B. 15 (1966) 627-637. doi:10.1002/pssb.19660150224.

[27] Q. Lu, X. Chen, S. Xie, X. Wu, Controllable and selective single-mode lasing in polymer microbottle resonator, Opt. Express. 26 (2018) 20183-20191. doi:10.1364/OE.26.020183.

[28] A. Bianchetti, A. Federico, S. Vincent, S. Subramanian, F. Vollmer, Refractometry-based air pressure sensing using glass microspheres as high-Q whispering-gallery mode microresonators, Opt. Commun. 394 (2017) 152-156. doi:10.1016/j.optcom.2017.03.009. 